\title{
Paper Performance Evaluation of MC-CDMA Systems with Single User Detection Technique using Kernel and Linear Adaptive Method
}

\author{
Rachid Fateh, Anouar Darif, and Said Safi \\ Sultan Moulay Slimane University, Beni Mellal, Morocco
}

https://doi.org/10.26636/jtit.2021.151621

\begin{abstract}
Among all the techniques combining multi-carrier modulation and spread spectrum, the multi-carrier code division multiple access (MC-CDMA) system is by far the most widely studied. In this paper, we present the performance of the MC-CDMA system associated with key single-user detection techniques. We are interested in problems related to identification and equalization of mobile radio channels, using the kernel method in Hilbert space with a reproducing kernel, and a linear adaptive algorithm, for MC-CDMA systems. In this context, we tested the efficiency of these algorithms, considering practical frequency selective fading channels, called broadband radio access network (BRAN), standardized for MC-CDMA systems. As far as the equalization problem encountered after channel identification is concerned, we use the orthogonality restoration combination (ORC) and the minimum mean square error (MMSE) equalizer techniques to correct the distortion of the channel. Simulation results demonstrate that the kernel algorithm is efficient for practical channels.
\end{abstract}

Keywords-BRAN channels, equalization, kernel method, MCCDMA, reproducing kernel Hilbert space.

\section{Introduction}

The problem of identifying a finite impulse response (FIR) system is the subject of interest for a large number of researchers [1]-[7]. Indeed, identifying a system means finding a set of parameters that form a mathematical model linking the different variables of the system [8]. Identification of linear systems has been carried out for decades using stochastic gradient algorithms. However, today, because of their complexity, the systems are becoming more non-linear. Given the increasing use of non-linear models in real systems, numerous resolution methods for the identification of non-linear systems, such as Volterra filters, neural networks and kernel methods, have been developed [9]-[14]. Kernel-based methods have been highly successful success in a wide range of fields over the past decade [15]. They are founded on the robust math- ematical framework of reproducing kernel Hilbert spaces (RKHS), creating an interesting framework for the development of adaptive non-linear filters [8], [16]. Concerns related to the application of these algorithms have led us to consider the problem of equalization of MC-CDMA systems using BRAN channels. In order to solve these issues, several authors have suggested additional approaches in [17]-[24]. Innovative multi-carrier CDMA [25] transmission techniques are emerging as high-potential solutions for the fourth-generation of cellular networks, due to the many advantages of MC-CDMA, such as reduced inter-symbol interference (ISI), excellent spectral efficiency, as well as the ability to prevent frequency-selective fading, multi-carrier modulation and demodulation using inverse fast Fourier transform (IFFT) at the transmitter and fast Fourier transform (FFT) at the receiver, as these are less complicated to implement. MC-CDMA has acquired a great deal of importance in multi-user wireless communication systems [26]. In this paper, the main goal is to analyze the performance of MC-CDMA systems used for downlink over BRAN channels. We considered two practical frequency-selective fading channels called broadband radio access network (BRAN C and BRAN E), standardized by the European Telecommunications Standards Institute (ETSI) [27], [28]. Performance of the downlink MC-CDMA systems' bit error rate (BER) using the BRAN measures is shown and compared with the results achieved with the kernel LMS and LMS algorithms.

The present paper is arranged as follows. In Section 2, we introduced the Hammerstein system identification architecture. In Section 3, derivation of the least mean square algorithm is presented. Section 4 reviews some basic concepts of the kernel methods, and the kernel least mean squares identification algorithm is presented. Section 5 depicts the model of the MC-CDMA system. Monte Carlo simulations of those algorithms are presented in Section 6 and, finally, Section 7 concludes the paper. 


\section{System Architecture}

Let us consider a Hammerstein model represented in Fig. 1. It consists of a non-linear static function followed by an invariant time linear block.

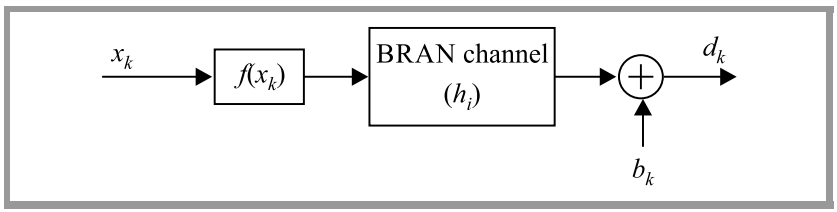

Fig. 1. Basic structure of a Hammerstein system with additive noise $b_{k}[9]$.

The system's output for a defined input signal $x_{k}$, where $k=0,1, \ldots$, is obtained as follows:

$$
d_{k}=\sum_{i=0}^{L-1} h_{i} f\left(x_{k-i}\right)+b_{k},
$$

where $x_{k}$ is the symbol emitted by the source at moment $k$, the channel impulse response $\left\{h_{i}\right\}_{i=0,1, \ldots, L-1}, L$ is the order of FIR system, and $f($.$) is the non-linearity.$

Throughout this paper, we rely on the following hypotheses concerning the system:

- input $x_{k}$, is independent and identically distributed (i.i.d.) with the zero mean.

- additive noise $b_{k}$ is Gaussian and independent of $x_{k}$ and $d_{k}$.

- non-linearity $f($.$) is continuous and invertible for any$ finite $x$.

- there is no delay in the system, i.e. $h_{0} \neq 0$.

\section{LMS Algorithm}

The least mean squares (LMS) [29] algorithm is one of the most popular algorithms for calculating coefficients of a finite impulse response filter. It is used to update the adaptive filter weights at each iteration:

$$
\omega_{n+1}=\omega_{n}+\mu_{L M S} e_{n} \mathbf{x}_{n},
$$

where $\mathbf{x}_{n}=\left[x_{n}, x_{n-1}, \ldots, x_{n-L+1}\right]^{\top}$ is the reference signal vector of length $L$ at moment $n, \omega_{n}=$ $\left.\omega_{0, n}, \omega_{1, n}, \ldots, \omega_{L-1, n}\right]^{\top} \in \mathbb{R}^{L}$ is the weight vector, $e_{n}=$ $d_{n}-y_{n}$ is the error between the desired signal and the output filter and $\mu_{L M S}$ is known as the algorithm's step-size parameter. Its value has an impact on the performance of the LMS algorithm. In order to ensure the convergence of the weighting vector, it is necessary for the convergence step to be included in the interval below [30]:

$$
0<\mu_{L M S}<\frac{2}{\lambda_{\max }}
$$

with $\lambda_{\max }$ being the maximum eigenvalue of the autocorrelation matrix, their values are non-negative.

The main disadvantage of this algorithm is the degradation of its performance when resolving non-linear problems.

\section{Kernel-Based Adaptive Filters}

Kernel methods undergo intense development these days, as they drive progress, both in terms of computational cost and performance achieved. They are based on a central principle known as the "kernel trick", exploited for the first time with the support vector machine (SVM) [31], adding a non-linear character to many originally linear methods. In practice, the kernel trick consists in rewriting an algorithm in which all relations between data inputs may be written as inner products, and replacing these products by scalar functions of two variables, where the original data input has been mapped to a non-linear Hilbert space (infinitedimensional) using the Mercers theorem [13]:

$$
\kappa\left(x_{i}, x_{j}\right)=\left\langle\psi\left(x_{i}\right), \psi\left(x_{j}\right)\right\rangle_{\mathcal{H}}, \quad \forall\left(x_{i}, x_{j}\right) \in \mathcal{E}^{2},
$$

where $\psi$ maps $\mathcal{E}$ into a higher dimensions space $\mathcal{H}$ with an inner product $\langle., .\rangle_{\mathcal{H}}$. Generally, $\operatorname{dim}(\mathcal{E}) \ll \operatorname{dim}(\mathcal{H})$.

In [13], [32]-[34], the authors have presented a detailed survey of the kernel method's characteristics. Figure 2 shows a functional diagram illustrating an adaptive filter based on the kernel, where $\left\{x_{n}\right\}_{1}^{N}$ is the vector of $N$ most recent input signal samples, $\left\{y_{n}\right\}_{1}^{N}$ is the estimated desired response, and $\left\{e_{n}\right\}_{1}^{N}$ is the estimation error.

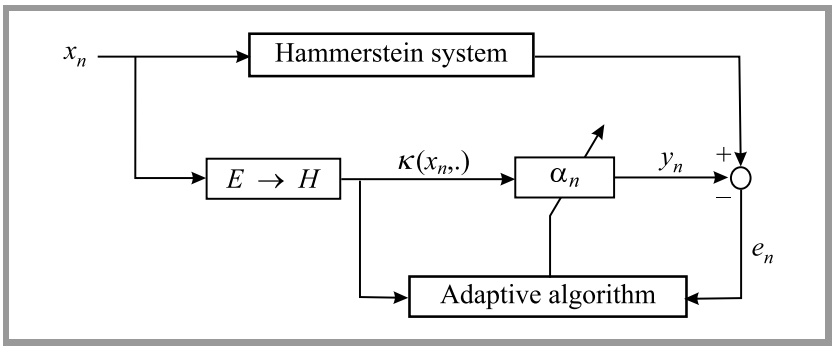

Fig. 2. Kernel-based adaptive system identification [35].

Any positively defined kernel can be seen as a scalar product in a functional space called the reproducing kernel Hilbert space (RKHS). This is therefore the property that a kernel must have to be validated. In order to properly determine the existence condition of a functional space $\mathcal{H}$, let us first start with some definitions [8], [36].

Definition 1 (positive definite kernel). A kernel is called positive definite if, for each input data point $\left\{x_{i}\right\}_{i=1}^{N} \in \mathcal{E}$, it satisfies the following condition:

$$
\alpha^{\top} K \alpha=\sum_{i, j=1}^{N} \alpha_{i} \alpha_{j} \kappa\left(x_{i}, x_{j}\right) \geq 0,
$$

for all $N \in \mathbb{N},\left(x_{1}, \ldots, x_{N}\right) \in \mathcal{E}^{N}$ and $\left(\alpha_{1}, \ldots, \alpha_{N}\right) \in \mathbb{R}^{N}$. We can assign an orthonormal base to a Hilbert space $\mathcal{H}$ in order to represent the elements of $\mathcal{H}$ from their coordinates. 
The associated kernel must be a continuous, symmetric, normalized and positive definite function $\kappa: \mathcal{E} \times \mathcal{E} \rightarrow \mathbb{R}$. $\mathcal{E}$ is the input domain, a compact subset of $\mathbb{R}^{N}$.

Definition 2 (reproducing kernels and Hilbert spaces). Let $\left(\mathcal{H},\langle.,\rangle_{\mathcal{H}}\right)$ represent a Hilbert space compromising functions of $\mathcal{E}$ in $\mathbb{R}$. The function $\kappa\left(x_{i}, x_{j}\right)$ of $\mathcal{E} \times \mathcal{E}$ in $\mathbb{R}$ is the reproducing kernel of $\mathcal{H}$, provided that $\mathcal{H}$ admits one, if there exists a function $\kappa(x,):. x_{i} \longrightarrow \kappa\left(x, x_{j}\right)$ belongs to $\mathcal{H}$, for any $x \in \mathcal{E}$.

\subsection{Kernel Least Mean Square}

Here, we present the LMS kernel algorithm [37]-[39]. The basic idea is to run the linear LMS algorithm specified by Eq. (2) in the kernel feature space which is associated with the positive defined kernel $\kappa$. The sequence of samples is transformed by means of a feature map:

$$
\begin{aligned}
\psi: & \mathcal{E} \longrightarrow \mathcal{H} \\
x & \longrightarrow \kappa(x, .) .
\end{aligned}
$$

To construct the RKHS model, we use the Gaussian kernel:

$$
\kappa\left(x_{i}, x_{j}\right)=\mathrm{e}^{-\frac{\left\|x_{i}-x_{j}\right\|^{2}}{2 \sigma^{2}}}, \quad \forall x_{i}, x_{j} \in \mathcal{E},
$$

where $\sigma>0$ is the width of the kernel.

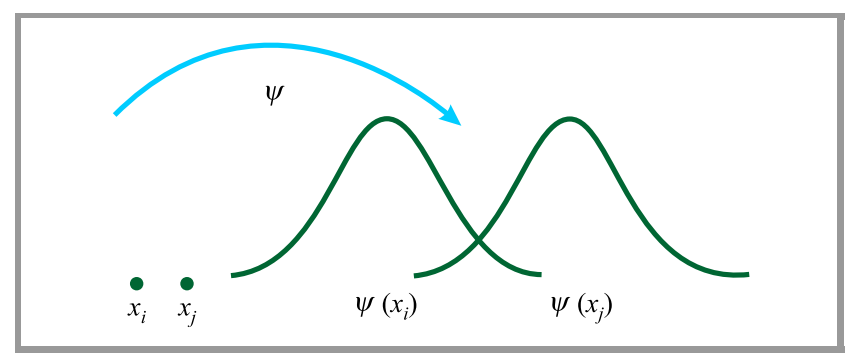

Fig. 3. Define a characteristic map.

Figure 3 shows data space $\mathcal{E}$ and space $\mathcal{H}$ induced by the kernel reproducing $\kappa$. This mapping represents each input point $x$ by its similarity $\kappa(x,$.$) to all other points in the \mathcal{E}$ domain. In order to build a feature space associated with $\psi$, the image of $\psi$ must be transformed into a vector space with an inner product [32]. Now let us assume that the sequence of samples is transformed using feature map $\psi$. The LMS logic may be applied on the following transformed data:

$$
\left\{\left(\psi\left(x_{1}\right), y_{1}\right),\left(\psi\left(x_{2}\right), y_{2}\right), \ldots\left(\psi\left(x_{n}\right), y_{n}\right), \ldots\right\} .
$$

As a consequence of the linear structure of the cost function $J_{\theta}(n)=E\left[\left(d_{n}-y_{n}\right)^{2}\right]$ can be minimized compared to $\theta$. We could solve this in the similar manner as in the LMS algorithm, using the instant-stochastic estimation, which gives:

$$
\hat{\theta}_{n+1}=\hat{\theta}_{n}+\mu e_{i} \psi\left(x_{i}\right)
$$

The main difference with LMS is that in Eq. (10) it is in a space of possibly infinite dimensional characteristics and its direct updating it would be virtually impossible. Instead, we will use each $\hat{\theta}_{n}$ of them to link to their initialization $\hat{\theta}_{0}$ :

$$
\hat{\theta}_{n}=\hat{\theta}_{0}+\mu \sum_{i=1}^{N} e_{i} \psi\left(x_{i}\right)
$$

By initializing the solution with a value 0 , the solution can be expressed, after $n$ iterations, as:

$$
\hat{\theta}_{n}=\mu \sum_{i=1}^{N} e_{i} \psi\left(x_{i}\right)
$$

By using the kernel trick, the following prediction function is obtained:

$$
\begin{aligned}
\left\langle\theta_{n}, \psi\left(x_{n}\right)\right\rangle_{\mathcal{H}} & =\mu \sum_{i=1}^{N} e_{i}\left\langle\psi\left(x_{i}\right), \psi\left(x_{n}\right)\right\rangle \\
& =\mu \sum_{i=1}^{N} e_{i} \kappa\left(x_{i}, x_{n}\right),
\end{aligned}
$$

where $\kappa\left(x_{i}, x_{n}\right)$ is a Mercer kernel, representing the inner product $\left\langle\psi\left(x_{i}\right), \psi\left(x_{n}\right)\right\rangle$ [39], and $N$ is the number of training samples.

\section{MC-CDMA System}

The MC-CDMA technique is based on concatenation of the spread spectrum and multi-carrier modulation [42]-[40]. Instead of applying the spread-spectrum technique in the time domain, we apply it in the frequency domain, by modulating the different chips of the spreading code with OFDM subcarriers. More precisely, the complex symbol $y_{j}$ specific to each user $j$ is first multiplied by its user-specific Walsh-Hadamard spreading code:

$C_{j}=\left(c_{u, j}\right)_{0<u<L_{c}}^{\top}$ of length $L_{c}$,

where (.) $)^{\top}$ designates the matrix transposition, then applied to the input of the multi-carrier modulator. Each sub-carrier transmits an information element multiplied by a chip of the specific code to this sub-carrier.

Figure 4 presents the general organization of a synchronous downlink based on the MC-CDMA technique in a scenario where length $L_{c}$ of the spreading code is equal to number $N_{c}$ of sub-carriers. In order to ensure orthogonality between subcarriers after the spreading function, the space between two adjacent subcarriers is proportional to the inverse of the $T_{c}$ duration of an MC-CDMA symbol on each subcarrier. For the $j$-th transmitter, the emitted signal was represented as:

$$
s_{j}(t)=\Re\left\{\frac{1}{\sqrt{N_{c}}} \sum_{k=0}^{N_{c}-1} y_{j} c_{j, k} \mathrm{e}^{\mathrm{i} 2 \pi f_{k} t}\right\} \text {, with } f_{k}=f_{0}+\frac{k}{T_{c}} .
$$

$f_{k}$ designates the relative frequency, $N_{c}$ corresponds to the number of subcarriers used, factor $\frac{1}{\sqrt{N_{c}}}$ is the power normalization term and $c_{j, k} \in\{-1,1\}$ denotes the spreading code. 


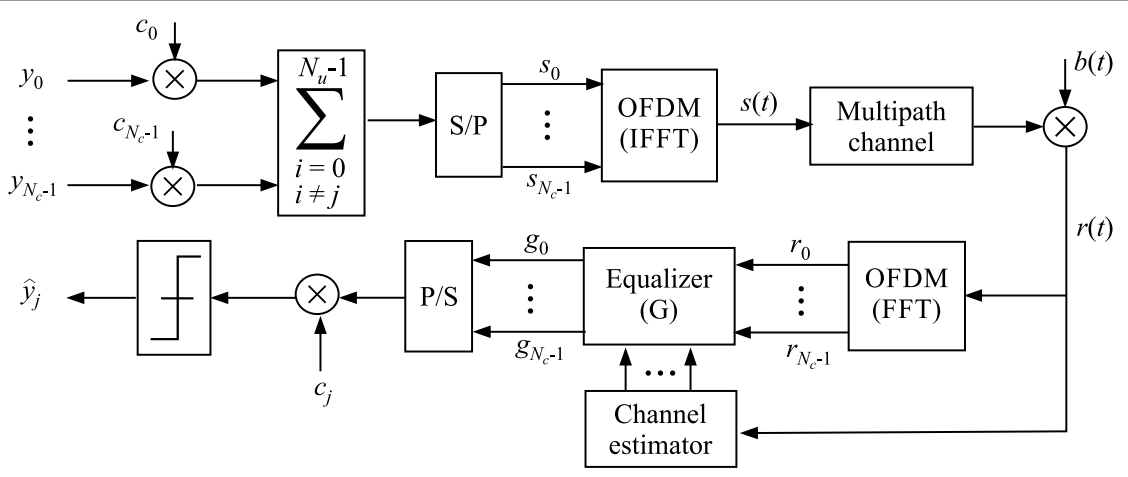

Fig. 4. MC-CDMA transmitter and receiver studied.

The propagation channel is time invariant and can be expressed in the delay domain by its impulse response:

$$
h(\tau)=\sum_{l=0}^{L-1} \beta_{l} \mathrm{e}^{\mathrm{j} \theta_{l}} \delta\left(\tau-\tau_{l}\right),
$$

where $\beta_{l}$ and $\theta_{l}$ are the magnitude and the phase of the channel, respectively.

The downlink MC-CDMA signal at the input of the receiver is noted $r(t)$ and is written as [43]:

$$
\begin{gathered}
r(t)=h(t) * s(t)+b(t) \\
r(t)=\frac{1}{\sqrt{N_{c}}} \sum_{l=0}^{L-1} \sum_{j=0}^{N_{u}-1} \sum_{p=0}^{N_{c}-1} \Re\left\{\beta_{l} \mathrm{e}^{\mathrm{j} \theta} y_{j} c_{j, p} \mathrm{e}^{2 \mathrm{j} \pi f_{k}\left(t-\tau_{l}\right)}\right\}+b(t)
\end{gathered}
$$

where $N_{u}$ is the number of users, $\mathfrak{R}$ is a function of the real part, and $b(t)$ is additive white Gaussian noise.

The MC-CDMA symbol received may be expressed in a vector form by:

$$
r=\left[r_{0}, r_{1}, \ldots, r_{N_{c}-1}\right]=H C y+b,
$$

where $r$ designates a vector made up of the values received on each subcarrier, and matrix $H$ represents the channel coefficients diagonal matrix:

$$
H=\left[\begin{array}{cccc}
h_{0} & 0 & \cdots & 0 \\
0 & h_{1} & \cdots & 0 \\
\vdots & \vdots & \ddots & \vdots \\
0 & 0 & \cdots & h_{N_{c}-1}
\end{array}\right]
$$

\subsection{Single-user Equalization for the MC-CDMA System}

The basic principle of equalization techniques is to reduce the effects of fading and interference, thus making it easier to make a decision about the received data symbols [17], [19]. Using the previous matrix notation, $G$, the diagonal matrix is composed of the $g_{j}$ equalization coefficients and can be expressed by:

$$
G=\left[\begin{array}{cccc}
g_{0} & 0 & \cdots & 0 \\
0 & g_{1} & \cdots & 0 \\
\vdots & \vdots & \ddots & \vdots \\
0 & 0 & \cdots & g_{N_{c}-1}
\end{array}\right]
$$

When equalized and de-spread according to sequence $c_{j}$ of the user under consideration, estimation $\hat{y}_{j}$ of the emitted symbol may be expressed as:

$$
\hat{y}_{j}=c_{j} \cdot G . r .
$$

Substituting Eq. (17) into Eq. (20) gives:

$$
\hat{y}_{j}=c_{j} . G . H . C . y+c_{j} . G \cdot b .
$$

Matrix $C$ represents $N_{c}$ spreading codes $c_{j}$ specific to each user $j$ :

$$
C=\left[\begin{array}{ccc}
c_{0,0} & \cdots & c_{0, N_{u}-1} \\
& & \\
c_{L_{c}-1,0} & \cdots & c_{N_{c}-1, L_{c}-1}
\end{array}\right],
$$

thus:

$$
\begin{aligned}
\hat{y}_{j}=\sum_{p=0}^{N_{c}-1} C_{p, j}^{2} g_{p} h_{p} y_{j} & +\sum_{\substack{q=0 \\
q \neq j}}^{N_{u}-1} \sum_{p=0}^{N_{c}-1} C_{p, j} C_{p, q} g_{p} h_{p} y_{q} \\
& +\sum_{p=0}^{N_{c}-1} C_{p, j} g_{p} b_{p} .
\end{aligned}
$$

Well-known single-user detection techniques include, inter alia, ORC and MMSE equalization.

\subsubsection{Orthogonality Restoration Combination (ORC)}

In order to completely cancel the phase and amplitude distortions provided by the channel, the ORC technique, also known as zero forcing (ZF), may be employed [23], [44]:

$$
g_{\text {orc }}=\frac{1}{\left|h_{p}\right|}, \quad \text { with } 0 \leq p \leq N_{c}-1 .
$$

Equation (23) allowing to obtain the estimation $\hat{y}_{j}$ of the symbol $y_{j}$ of the user $j$ is then written as:

$$
\begin{aligned}
\hat{y}_{j}=\sum_{p=0}^{N_{c}-1} C_{p, j}^{2} y_{j}+ & \sum_{\substack{q=0 \\
q \neq j}}^{N_{u}-1} \sum_{p=0}^{N_{c}-1} C_{p, j} C_{p, q} y_{q} \\
& +\sum_{p=0}^{N_{c}-1} C_{p, j} \frac{1}{h_{p}} b_{p} .
\end{aligned}
$$


Assuming that the spreading codes used at the transmitter level are orthogonal, we have:

$$
\sum_{p=0}^{N_{c}-1} C_{p, j} C_{p, q}=0, \quad \forall j \neq q .
$$

The Eq. (25) reduces to:

$$
\hat{y}_{j}=\sum_{p=0}^{N_{c}-1} C_{p, j}^{2} y_{j}+\sum_{p=0}^{N_{c}-1} C_{p, j} \frac{1}{h_{p}} b_{p} .
$$

However, the main drawback of this technique is that, for weak amplitudes of $h_{p}$, the multiplication by an inverse function of the channel results in a strong amplification of the noise, which rapidly increases the value of $g_{\text {orc }}$.

\subsubsection{Minimum Mean Square Error (MMSE)}

Equalization performed according to the MMSE criterion aims to minimize the mean square value of the error between vector $S$ of the transmitted signal and its estimate $\hat{S}=G R$ generated at the output of the equalizer:

$$
J=E\left\{|\varepsilon|^{2}\right\}=E\left\{|S-G R|^{2}\right\},
$$

where $R=\left(R_{0}, \ldots, R_{k}, \ldots, R_{N_{c}-1}\right)$ and $G=\left(G_{0}, \ldots\right.$, $\left.G_{k}, \ldots, G_{N_{c}-1}\right)$ are the symbol vector returned at the output of the FFT and the gain vector of the equalizer, respectively [45], [23]. This mean squared error $J$ is minimal when the equalizer's $G_{k}$ gains are such that both the received signal and the error signal are orthogonal, meaning that:

$$
E\left\{\varepsilon R^{* \top}\right\}=0 \text {. }
$$

When the number of users is equal to length $L_{c}$ of the code, the optimal equalizer coefficients, according to the mean square error minimization criterion, are:

$$
g_{\text {mmse }}=\frac{h_{p}^{*}}{\left|h_{p}\right|^{2}+\frac{1}{\gamma_{p}}}, \quad \text { with } \quad \gamma_{p} \neq 0,
$$

where $g_{\text {mmse, } p}$ is the $p$-th complex channel gain, the operation $*$ is the complex conjugate and $\gamma_{p}$ is the signal-to-noise ratio for subcarrier $p$. The estimated received symbol, $\hat{y}_{i}$ of the symbol $y_{i}$ of user $i$ is represented by:

$$
\begin{array}{r}
\hat{y}_{j}=\underbrace{\sum_{p=0}^{N_{c}-1} C_{p, j}^{2} \frac{\left|h_{p}\right|^{2}}{\left|h_{p}\right|^{2}+\frac{1}{\gamma_{p}}} y_{j}}_{\lambda}+\underbrace{\sum_{q=0}^{N_{u}-1} \sum_{p=0}^{N_{c}-1} C_{p, j} C_{p, q} \frac{\left|h_{p}\right|^{2}}{\left|h_{p}\right|^{2}+\frac{1}{\gamma_{p}}} y_{p}}_{\varsigma(\mathrm{q} \neq \mathrm{j})} \\
+\underbrace{\sum_{p=0}^{N_{c}-1} C_{p, j} \frac{h_{p}^{*}}{\left|h_{p}\right|^{2}+\frac{1}{\gamma_{p}}} b_{p}}_{\eta}, \quad \text { (30) }
\end{array}
$$

where $\lambda$ is the usable signal portion, $\varsigma$ is the multiple access interference and $\eta$ in the noise term.

If we assume that the code for spreading is orthogonal, that is:

$$
\sum_{p=0}^{N_{u}-1} C_{p, j} C_{p, q}=0, \quad \forall j \neq p
$$

So, Eq. (30) becomes:

$$
\hat{y}_{j}=\sum_{p=0}^{N_{u}-1} C_{p, j}^{2} \frac{\left|h_{p}\right|^{2}}{\left|h_{p}\right|^{2}+\frac{1}{\gamma_{p}}} y_{j}+\sum_{p=0}^{N_{u}-1} C_{p, j} \frac{h_{p}^{*}}{\left|h_{p}\right|^{2}+\frac{1}{\gamma_{p}}} b_{p}
$$

\section{Simulation and Results}

The simulation will allow us to study the performance of the adaptive kernel filtering algorithm. The mean square error (MSE) will be used to measure the accuracy of the estimated values as:

$$
\operatorname{MSE}(h, \hat{h})=\frac{1}{p} \sum_{i=1}^{p}\left[\frac{h(i)-\hat{h}(i)}{h(i)}\right]^{2},
$$

where $h(i)$ is the measured impulse response, $\hat{h}(i)$ is the estimated impulse response, and $p$ represents the length of the impulse response.

MC-CDMA system's equalization performance in the single-user downlink scenario is evaluated using MMSE and ORC equalizers. This assessment is carried out using measured and estimated parameters of practical broadband radio access network (BRAN $\mathrm{C}$ and BRAN E) models to determine the bit error rate (BER), applying KLMS and LMS algorithms.

\subsection{BRAN C and BRAN E Parameters}

In Tables 1 and 2, we represent the measured values corresponding to BRAN C and BRAN E radio channels' impulse response. The impulse response of each model is:

$$
h(n)=\sum_{i=1}^{p} M_{i} \delta\left(n-\tau_{i}\right)
$$

where $\delta(n)$ is the Dirac's function, $\tau_{i}$ is path's $i$ time delay, $M_{i}$ is path's $i$ magnitude, where their impulse responses are between 0 and $1, M_{i} \in N(0,1), i=1, \ldots, p$ and $p=18$ is the number of paths.

Table 1

Delay and magnitudes of 18 targets of a BRAN C channel

\begin{tabular}{|c|c||c|c|}
\hline $\begin{array}{c}\text { Delay } \\
\tau_{i}[\mathrm{~ns}]\end{array}$ & $\begin{array}{c}\text { Magnitude } \\
M_{i}[\mathrm{~dB}]\end{array}$ & $\begin{array}{c}\text { Delay } \\
\tau_{i}[\mathrm{~ns}]\end{array}$ & $\begin{array}{c}\text { Magnitude } \\
M_{i}[\mathrm{~dB}]\end{array}$ \\
\hline 0 & -3.3 & 230 & -3.0 \\
10 & -3.6 & 280 & -4.4 \\
20 & -3.9 & 330 & -5.9 \\
30 & -4.2 & 400 & -5.3 \\
50 & 0 & 490 & -7.9 \\
80 & -0.9 & 600 & -9.7 \\
110 & -1.7 & 730 & -13.2 \\
140 & -2.6 & 880 & -16.3 \\
180 & -1.5 & 1050 & -21.2 \\
\hline
\end{tabular}

Table 3 shows the various parameters of the MC-CDMA system implemented for the two BRAN channels: $\mathrm{C}$ and $\mathrm{E}$. 
Table 2

Delay and magnitudes of 18 targets of a BRAN E channel

\begin{tabular}{|c|c||c|c|}
\hline $\begin{array}{c}\text { Delay } \\
\tau_{i}[\mathrm{~ns}]\end{array}$ & $\begin{array}{c}\text { Magnitude } \\
M_{i}[\mathrm{~dB}]\end{array}$ & $\begin{array}{c}\text { Delay } \\
\tau_{i}[\mathrm{~ns}]\end{array}$ & $\begin{array}{c}\text { Magnitude } \\
M_{i}[\mathrm{~dB}]\end{array}$ \\
\hline 0 & -4.9 & 320 & 0 \\
10 & -5.1 & 430 & -1.9 \\
20 & -5.2 & 560 & -2.8 \\
40 & -0.8 & 710 & -5.4 \\
70 & -1.3 & 880 & -7.3 \\
100 & -1.9 & 1070 & -10.6 \\
140 & -0.3 & 1280 & -13.4 \\
190 & -1.2 & 1510 & -17.4 \\
240 & -2.1 & 1760 & -20.9 \\
\hline
\end{tabular}

Table 3

Simulation parameters

\begin{tabular}{|l|c|}
\hline Characteristic parameters & Configuration \\
\hline Spreading code lengths $L_{c}$ & 64 \\
Sampling frequency $f_{s}$ & $20 \mathrm{MHz}$ \\
Number of sub-carriers $N_{c}$ & 64 \\
Symbol times $T_{s}$ & $3 \mu \mathrm{s}$ \\
Number of users $N_{u}$ & 64 \\
Spreading codes & Walsh-Hadamard \\
OFDM modulation & FFT 64 samples \\
Channel model & BRAN (C and E) \\
Performance metrics & BER and MSE \\
\hline
\end{tabular}

\subsection{Performance Results}

\subsubsection{ETSI BRAN C Channel}

The parameters of the impulse response ETSI BRAN C radio channel estimated using the two algorithms are presented in Fig. 5, for a scenario with $S N R=15 \mathrm{~dB}$, the number of input signal samples fixed at $N=1024$ and 50 Monte Carlo iterations used. One may notice that the response estimated using the kernel algorithm matches the real values,

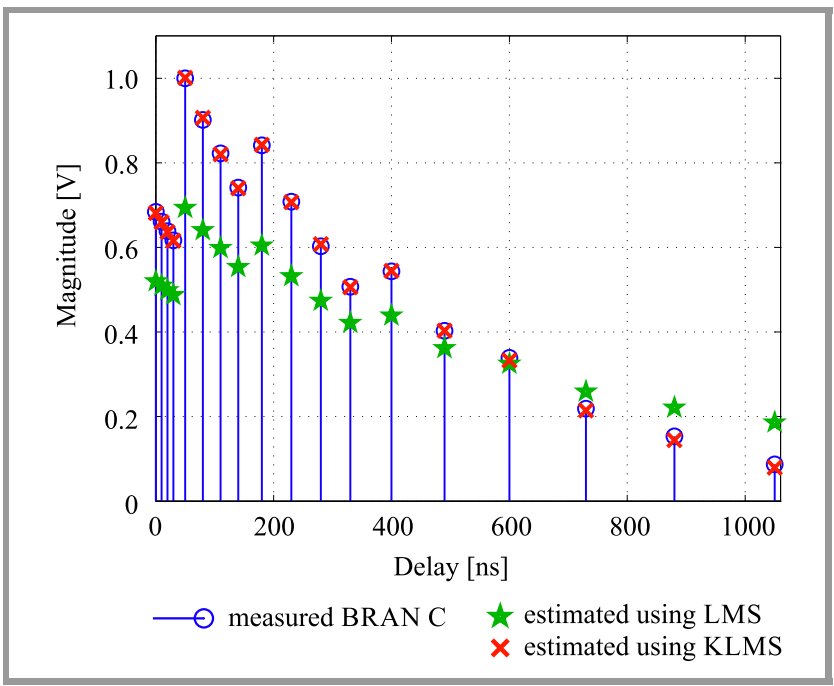

Fig. 5. Estimate of the BRAN C channel amplitude depending on the delay time. but when estimating the channel impulse response using the LMS algorithm, there is an apparent difference between the estimated and the measured values.

The mean square error values for the two algorithms are presented in Fig. 6, for an SNR varying between $0 \mathrm{~dB}$ and $40 \mathrm{~dB}$ and the number of samples equaling $N=1024$. We find that the kernel algorithm offers the best performance in terms of MSE criteria even in a highly noisy environment, compared to the LMS algorithm.

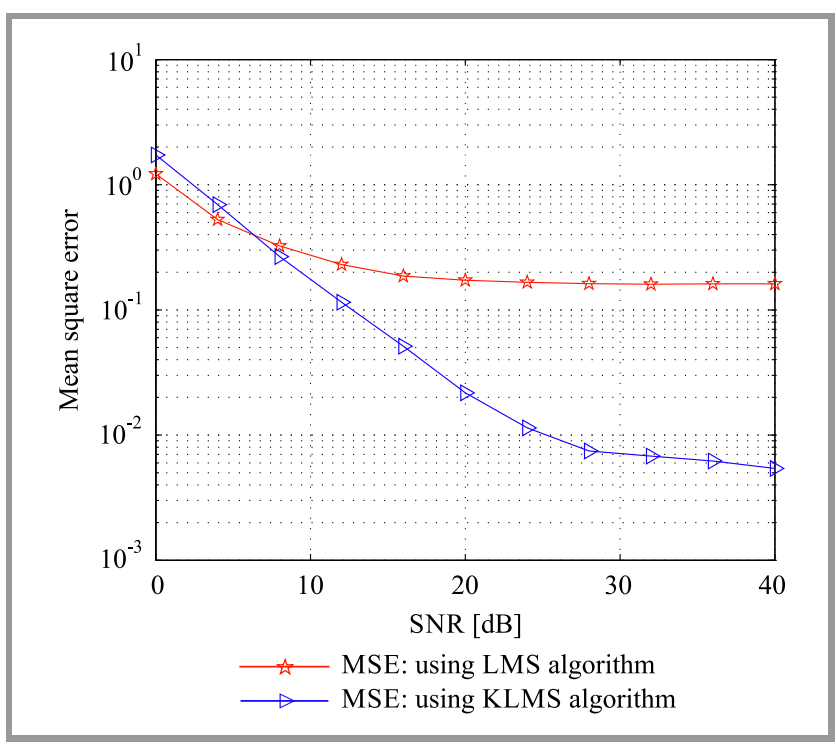

Fig. 6. Comparison of algorithms in terms of MSE for different $S N R$ values and for the data length of $N=1024$, BRAN C channel.

Figure 7 demonstrates estimates concerning of the amplitude and phase of the BRAN C channel, using the least mean square and the kernel least mean square algorithms, for a number of samples equaling $N=1024$ and for an $S N R=15 \mathrm{~dB}$. These results allow us to conclude that the kernel algorithm is more efficient compared with the LMS algorithm, because it allows us to have the same paces of the estimated (amplitude and phase) and those measured.

Figures 8 and 9 show, respectively, the BER simulation results, in the single-user and in the downlink scenario, for different SNRs obtained using ORC and MMSE equalizers, based on the measured parameters of the BRAN C channel and parameters estimated by the KLMS and LMS algorithms. From these simulation results (Fig. 8), one may conclude that the KLMS algorithm predicts the same performance values as those measured for the BRAN $\mathrm{C}$ channel, as opposed to the LMS algorithm which yields values quite different from their measured counterparts (BRAN C). Indeed, the Kernel LMS algorithm's BER values are inferior to those achieved by the LMS algorithm. From Figs. 8 and 9, we can conclude that:

- when using ORC equalization measured values. Both the two algorithms give the 1 bit error if we receive $10^{2}$ bits for $S N R=24 \mathrm{~dB}$, with an advantage over the kernel algorithm (Fig. 8), 


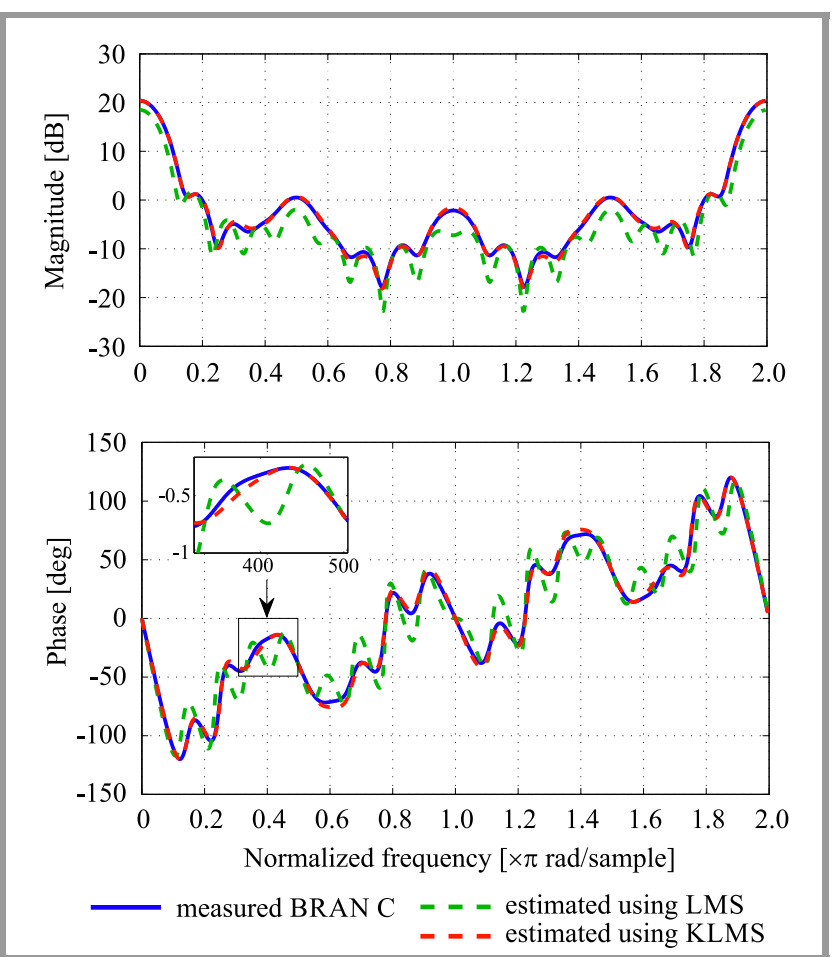

Fig. 7. Estimation of the amplitude and phase of the BRAN C channel, for the data length of $N=1024$ and $S N R=15 \mathrm{~dB}$.

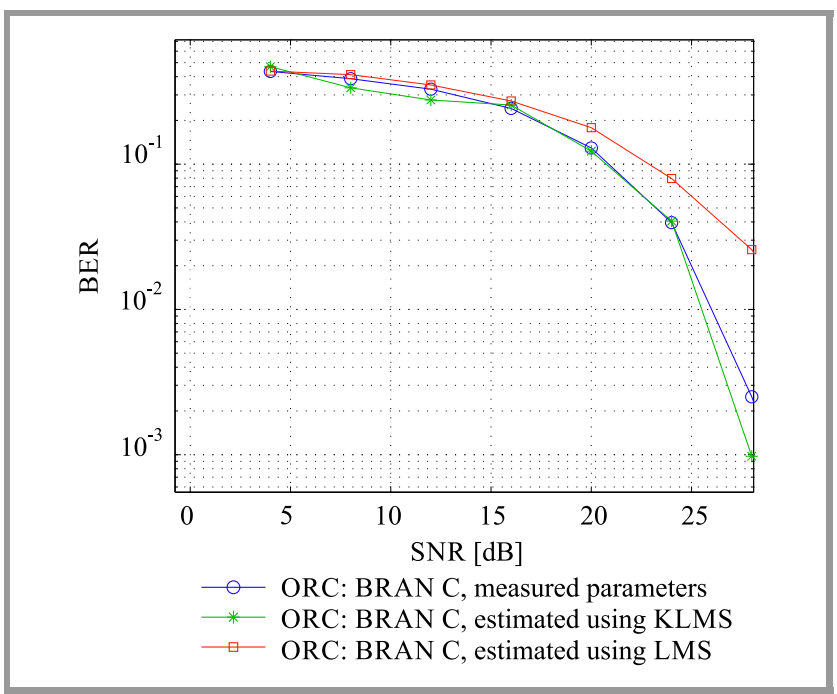

Fig. 8. BER vs. SNR of the estimated and measured BRAN C channel, using the ORC equalizer.

- in the case of the MMSE equalizer, if $S N R=24 \mathrm{~dB}$ we just get a 1 error bit, when we receive $10^{4}$ bits, with an advantage over the kernel algorithm (Fig. 9).

We conclude that the MMSE technique is more efficient than the ORC technique in the case of the ETSI BRAN C radio channel.

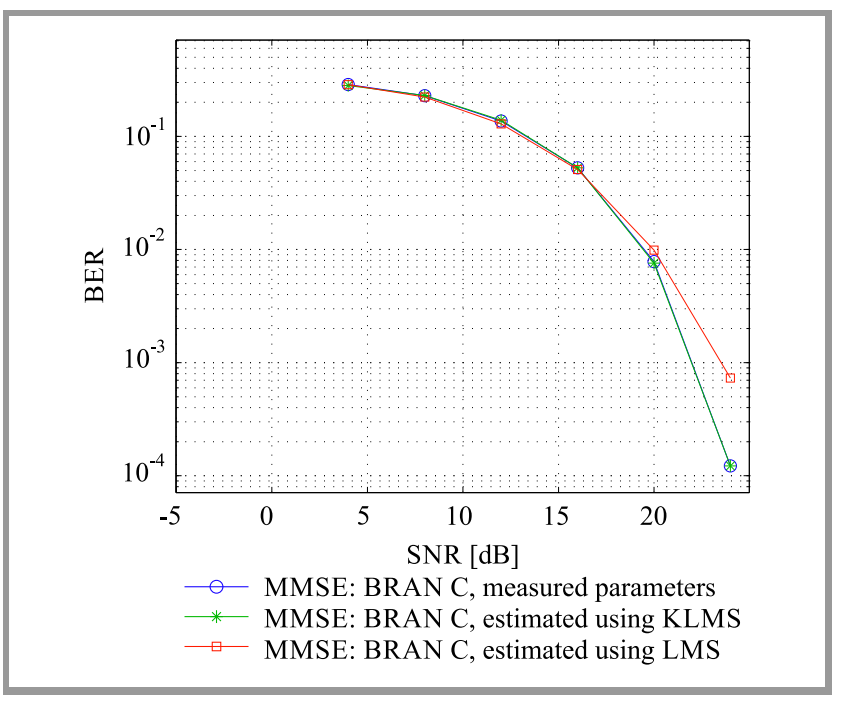

Fig. 9. BER vs. SNR of the estimated and measured BRAN C channel, using the MMSE equalizer.

\subsubsection{ETSI BRAN E Channel}

Figure 10 shows the estimated parameters of the BRAN E channel impulse response, as a function of the path delays, for a data length of $N=1024, S N R=15 \mathrm{~dB}$, and 50 Monte Carlo runs. One may notice that the best performance is obtained by the kernel LMS algorithm.

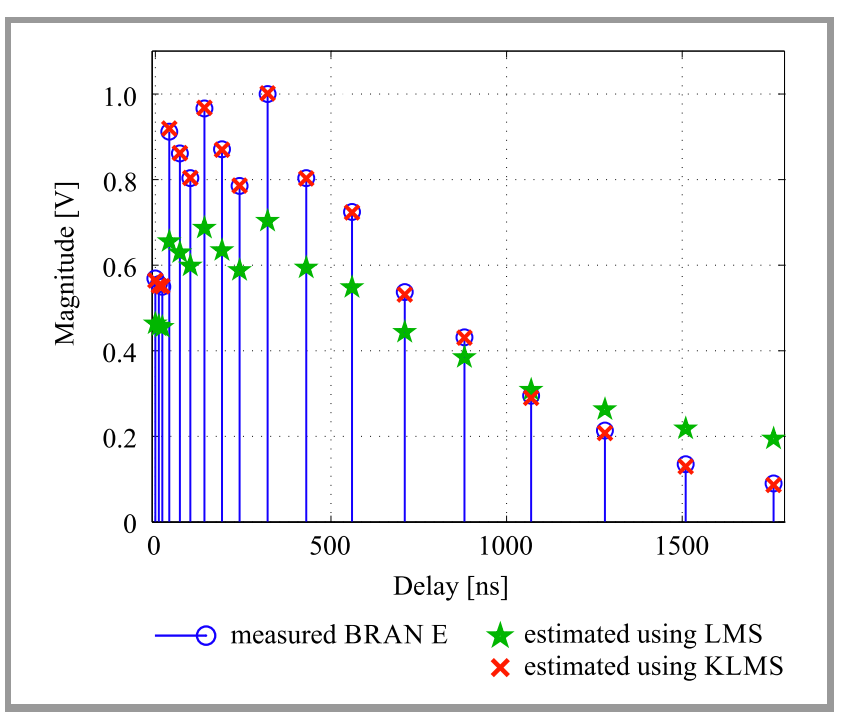

Fig. 10. BRAN E channel amplitude estimates depending on the time delay.

The mean square error values for the two algorithms are represented in Fig. 11, for different SNR and for the numbers of samples equaling $N=1024$. One may notice that the performance of LMS is almost unaffected by any considerable disturbance, whereas the performance of the kernel has been significantly reduced due to its sensitivity to low SNR. The kernel algorithm achieves the best performance, with higher convergence speed and minimized MSE. 


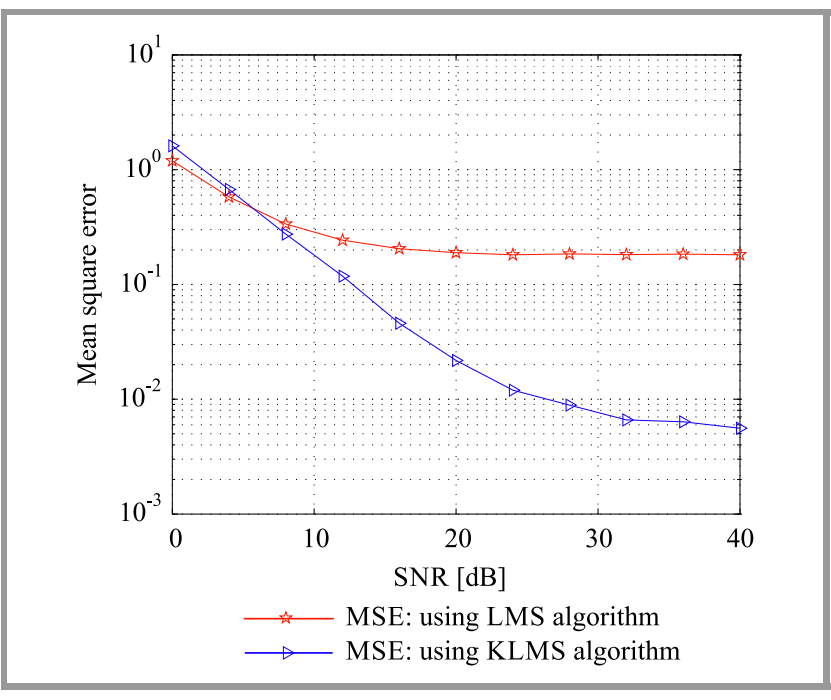

Fig. 11. Comparison of algorithms in terms of MSE for different $S N R$ values and $N=1024$, BRAN E channel.

As summarized in Figs. 11 and 6, it can be seen that the LMS algorithm is more effective in the case of the BRAN $C$ channel, due to the greater level of fluctuations in the BRAN E channel. On the basis of the obtained results, one may notice that when the $S N R$ value is lower than $5 \mathrm{~dB}$, the value of MSE obtained with the KLMS algorithm (MSE $>10^{\circ}$, if $S N R=0 \mathrm{~dB}$ ) is slightly higher than the one obtained with LMS (MSE $=10^{\circ}$, if $\left.S N R=0 \mathrm{~dB}\right)$, but when $S N R>5 \mathrm{~dB}$, we note the stability of the MSE value of the LMS so that no $S N R$ value was affected, which testifies
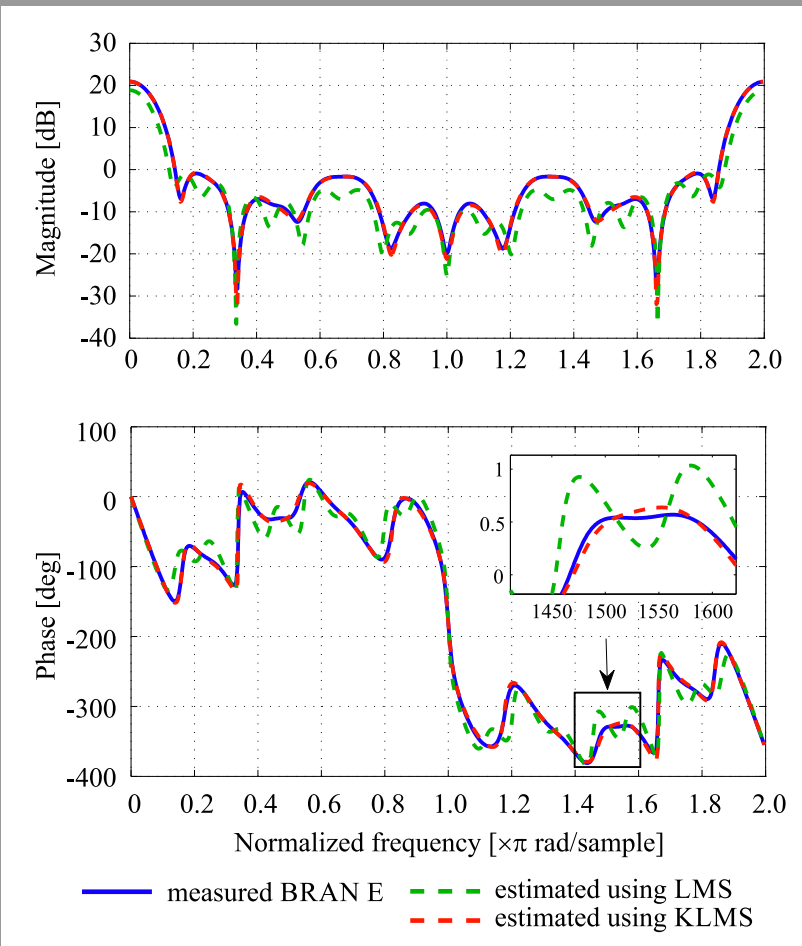

Fig. 12. Estimation of the amplitude and phase of the BRAN E channel, for a data length of $N=1024$ and $S N R=15 \mathrm{~dB}$. to its incapacity to estimate the parameters. These results allow us to conclude that the kernel algorithm gives a good approximation of the model parameters to be identified. The estimation of the amplitude and phase of the BRAN E channel, using the two algorithms, is presented in Fig. 12, for a number of samples $N=1024$ and $S N R=15 \mathrm{~dB}$. The amplitude and phase estimated using the kernel algorithm have the same form as the measured data. Compared to the linear adaptive algorithm (LMS), we notice a difference between the shape of estimated amplitude and phase, and the shape of the actually measured parameters.

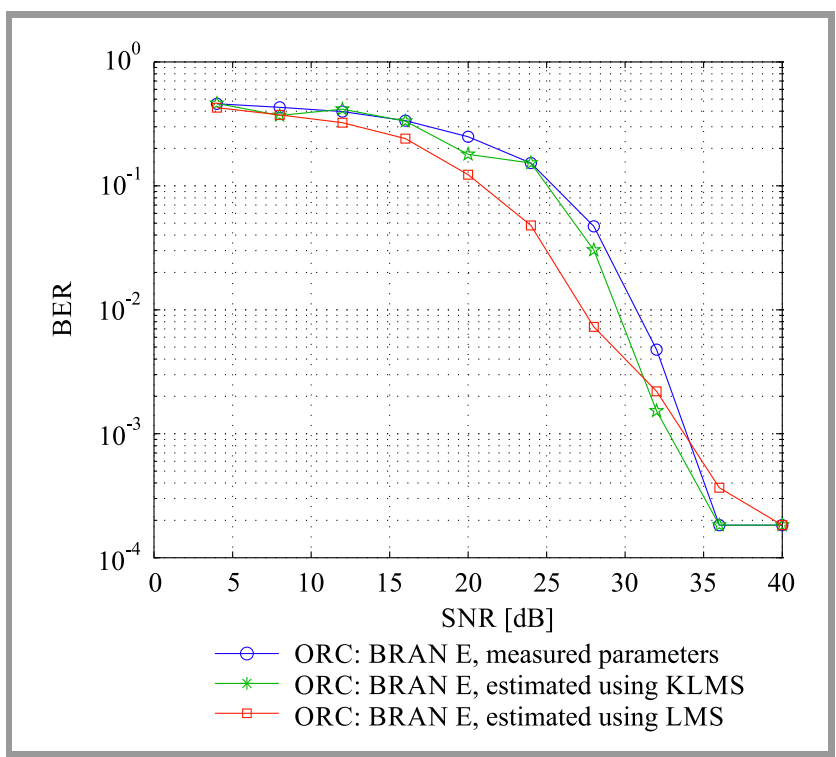

Fig. 13. BER vs. SNR of the estimated and measured BRAN E channel, using ORC equalizer.

The plot shown in Fig. 13 indicates BER for various SNRs, achieved using the kernel algorithm, and compares with the

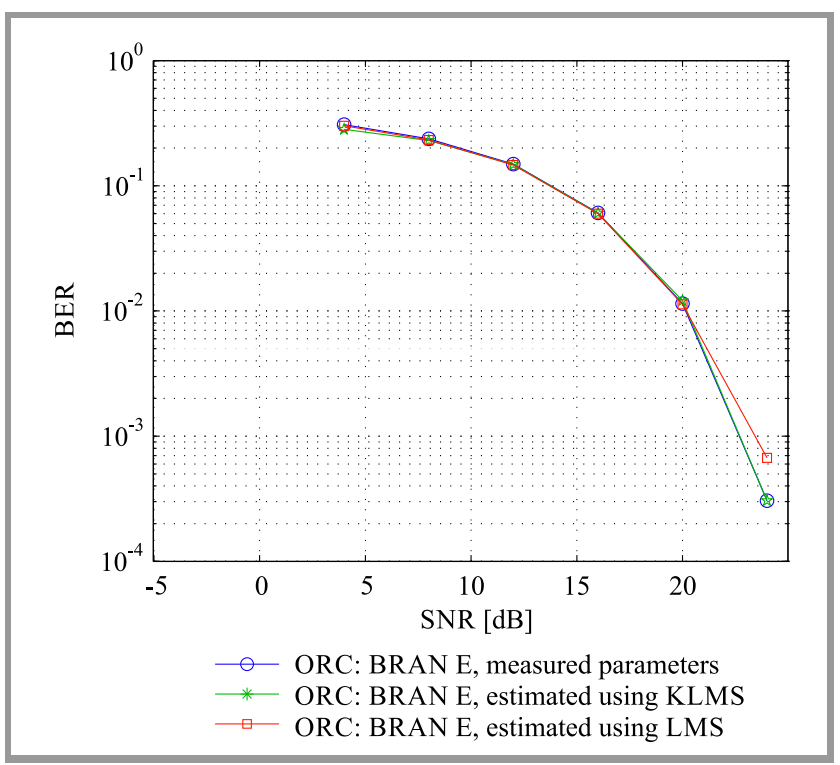

Fig. 14. BER vs. SNR of the estimated and measured BRAN E channel, using the MMSE equalizer. 
values obtained with the LMS algorithm. Equalization is performed using the ORC equalizer.

Similarly, Fig. 14 presents the results of BER simulations for different SNRs obtained using the MMSE equalizer, based on the measured parameters of the BRAN E channel and the ones estimated with KLMS and LMS algorithms. We note that the efficiency of the KLMS algorithm is important compared to the LMS algorithm. According to Figs. 13 and 14 , if $S N R=36 \mathrm{~dB}$, the BER value is lower than $10^{-3}$ in the case of the ORC equalizer, but when using the MMSE technique, we obtain a BER value that is lower than $10^{-3}$ when the $S N R$ equals $24 \mathrm{~dB}$.

One may conclude that the MMSE equalizer offers the best performance in terms of BER for all channels studied (BRAN C and BRAN E).

\section{Conclusion}

In this paper, the performance of an MC-CDMA system in the downlink over BRAN channels was evaluated and analyzed, using the kernel LMS and LMS algorithms. These algorithms are used for estimating the parameters of the measured channels in different scenarios (BRAN $\mathrm{C}$ and BRAN E). The results presented in the identification part demonstrate that the kernel algorithm is effective and efficient for practical channels. As far as equalization of MCCDMA systems is concerned, we obtained excellent bit error rate performance, mainly if the kernel last mean square algorithm was used. Future research includes the development of an extension of these algorithms to MIMO systems, new methods for identifying communication channels (i.e. using kernel methods) and development of new equalizers practically for MC-CDMA systems can be presented in the future.

\section{References}

[1] M. Pouliquen, T. Menard, E. Pigeon, O. Gehan, and A. Goudjil, "Recursive system identification algorithm using binary measurements", in European Control Conference (ECC), Aalborg, Denmark, 2016, pp. 1353-1358 (DOI: 10.1109/ECC.2016.7810477).

[2] M. Zidane, S. Safi, M. Sabri, and A. Boumezzough, "Impulse response identification of minimum and non minimum phase channels", Int. J. of Adv. Sci. and Technol., vol. 64, 2014, pp. 59-72 (DOI: 10.14257/ijast.2014.64.06).

[3] U. Soverini and T. Söderström, "Frequency domain identification of FIR models in the presence of additive input-output noise", Automatica, vol. 115, pp. 108879, 2020 (DOI: 10.1016/j.automatica.2020.108879).

[4] H. Zhang, T. Wang, and Y. Zhao, "Asymptotically efficient recursive identification of FIR systems with binary-valued observations", IEEE Trans. on Systems, Man, and Cybernetics: Systems, vol. 51, no. 5, pp. 2687-2700, 2021 (DOI: 10.1109/TSMC.2019.2916022).

[5] U. Soverini and T. Söderström, "Frequency domain identification of ARX models in the presence of additive input-output noise", IFACPapersOnLine, vol. 50, no. 1, pp. 6226-6231, 2017 (DOI: 10.1016/j.ifacol.2017.08.1023).

[6] R. Fateh and A. Darif, "Mean square convergence of reproducing kernel for channel identification: application to BRAN D channel impulse response", in Int. Conf. on Business Intell., Springer: Cham, Switzerland, 2021, pp. 284-293 (DOI: 10.1007/978-3-030-76508-8_20).
[7] R. Fateh, A. Darif, and S. Safi, "Channel Identification of Non-linear Systems with Binary-Valued Output Observations Based on Positive Definite Kernels", in E3S Web of Conferences, EDP Sciences, vol. 297, pp. 01020, 2021 (DOI: 10.1051/e3sconf/202129701020).

[8] S. Van Vaerenbergh, "Kernel methods for nonlinear identification, equalization and separation of signals", Ph.D. dissertation, Universidad de Cantabria, Santander, Spain, 2010 [Online]. Available: https://gtas.unican.es/files/pub/tesis_Steven.pdf

[9] R. Castro Garcia, "Structured nonlinear system identification using kernel-based methods", Ph.D. dissertation, Faculty of Engineering Science, KU Leuven, Belgium, 2017 [Online]. Available: https://lirias.kuleuven.be/retrieve/473580

[10] S. Gupta, A. K. Sahoo, and U. K. Sahoo, "Volterra and Wiener model based temporally and spatio-temporally coupled nonlinear system identification: a synthesized review", IETE Technical Review, 2020, pp. 1-25 (DOI: 10.1080/02564602.2020.1732233).

[11] F. Pérez-Cruz and O. Bousquet, "Kernel methods and their potential use in signal processing”, IEEE Signal Process. Mag., vol. 21, no. 3, pp. 57-65, 2004 (DOI: 10.1109/MSP.2004.1296543).

[12] W. Liu, J. C. Principe, and S. Haykin, "Kernel adaptive filtering: a comprehensive introduction", vol. 57, John Wiley \& Sons, 2011 (ISBN: 9780470447536).

[13] N. Aronszajn, "Theory of reproducing kernels", Trans. of the American mathematical society, vol. 68, no. 3, pp. 337-404, 1950 (DOI: 10.2307/1990404).

[14] S. Van Vaerenbergh and L. A. Azpicueta-Ruiz, "Kernel-based identification of Hammerstein systems for nonlinear acoustic echocancellation", in IEEE Int. Conf. on Acoustics, Speech and Signal Process. (ICASSP), Florence, Italy, 2014. pp. 3739-3743 (DOI: 10.1109/ICASSP.2014.6854300).

[15] C. Richard, J. C. M. Bermudez, and P. Honeine, "Online Prediction of Time Series Data With Kernels", IEEE Trans. on Signal Process., vol. 57, no. 3, pp. 1058-1067, 2009 (DOI: 10.1109/TSP.2008.2009895).

[16] O. A. Arqub and H. Rashaideh, "The RKHS method for numerical treatment for integrodifferential algebraic systems of temporal twopoint BVPs", Neural Computing and Applications, vol. 30, no. 8, pp. 2595-2606, 2018 (DOI: 10.1007/s00521-017-2845-7).

[17] M. Boutalline, J. Antari, M. Gouskir, B. Bouikhalene, and S. Safi, "A robust approach for Proakis and BRAN channel identification and equalization of MC-CDMA systems", in 5th Int. Conf. on Optimization and App. (ICOA), Kenitra, Morocco, 2019, pp. 1-9 (DOI: 10.1109/ICOA.2019.8727665).

[18] S. Safi, M. Frikel, M. Pouliquen, I. Badi, Y. Khmou, and M. Boutalline, "MC-CDMA system identification and equalization using the LMS algorithm and Takagi-Sugeno fuzzy system", IFAC Proc. Volumes, vol. 46, no. 11, pp. 599-604, 2013 (DOI: 10.3182/20130703-3-FR-4038.00027).

[19] M. Zidane, S. Safi, M. Sabri, and A. Boumezzough, "Blind identification channel using higher order cumulants with application to equalization for MC-CDMA system", Int. J. of Elec., Robot., Electron. and Commun. Engin., vol. 8, no. 2, pp. 369-375, 2014 (DOI: 10.5281/zenodo.1091694).

[20] M. S. Sakib and M. R. Ferdous, "Performance evaluation of MC-CDMA system for the next generation wireless network", in Int. Conf. on Innovation in Engin. and Technol. (ICIET), Dhaka, Bangladesh, 2018, pp. 1-5 (DOI: 10.1109/CIET.2018.8660783).

[21] M. Zidane, S. Safi, M. Sabri, A. Boumezzough, and M. Frikel, "Broadband radio access network channel identification and downlink MC-CDMA equalization", Int. J. of Energy, Inform. and Commun., vol. 5, no. 2, pp. 13-34, 2014 (DOI: 10.14257/ijeic.2014.5.2.02).

[22] S. Safi, M. Frikel, M. M'Saad, and A. Zeroual, "Blind identification and equalization of downlink TCM coded MC-CDMA systems using cumulants", in 16th European Signal Process. Conf., IEEE. Lausanne, Switzerland, 2008, pp. 1-5, 2008 (ISSN: 22195491).

[23] M. Zidane, Mohammed, S. Safi, and M. Sabri, "Measured and estimated data of non-linear BRAN channels using HOS in 4G wireless communications", Data in brief, vol. 17, pp. 1136-1148, 2018 (DOI: 10.1016/j.dib.2018.02.005). 
[24] M. Zidane, S. Safi, and M. Sabri, "Compensation of fading channels using partial combining equalizer in MC-CDMA systems", $J$. of Telecommun. and Informat. Technol., no. 1, pp. 5-11, 2017 [Online]. Available: http://dlibra.itl.waw.pl/ dlibra-webapp/Content/1962/ISSN_1509-4553_1_2017_5.pdf

[25] S. Chitra, and N. Kumaratharan, "Multimedia transmission in MCCDMA using adaptive subcarrier power allocation and CFO compensation", Int. J. of Electron., vol. 105, no. 2, pp. 289-302, 2018 (DOI: 10.1080/00207217.2017.1357201).

[26] S. Hara and R. Prasad, "Overview of multicarrier CDMA", IEEE Commun. Mag., vol. 35, no. 12, pp. 126-133, 1997 (DOI: 10.1109/35.642841).

[27] "Broadband radio access networks (BRAN); High performance radio logical area network (HIPERLAN) Type 2; Requirements and architectures for wireless broadband access", ETSI, 1999 [Online]. Available: https://www.etsi.org/deliver/etsi_tr/101000_101099/ 101031/02.02.01_60/tr_101031v020201p.pdf

[28] "Broadband radio access networks (BRAN); HIPERLAN Type 2; Physical layer", ETSI, 2001 [Online]. Available: https://www.etsi.org/deliver/etsi_ts/101400_101499/ 101475/01.02.02_60/ts_101475v010202p.pdf

[29] R. Martinek, J. Rzidky, R. Jaros, P. Bilik, and M. Ladrova, "Least mean squares and recursive least squares algorithms for total harmonic distortion reduction using shunt active power filter control", Energies, vol. 12, no. 8, pp. 1545, 2019 (DOI: 10.3390/en12081545).

[30] S. Haykin, "Adaptive filter theory (4-th edition)", Prentice Hall, 2001 (ISBN: 9780130901262).

[31] C. Cortes and V. Vapnik, "Support-vector networks", Machine Learning, vol. 20, no. 3, pp. 273-297, 1995 (DOI: 10.1007/BF00994018).

[32] B. Schölkopf, A. J. Smola, and F. Bach, "Learning with Kernels: Support vector machines, regularization, optimization, and beyond", MIT Press, 2002 [Online]. Available: https://alex.smola.org/papers/2001/SchHerSmo01.pdf

[33] B. Schölkopf, H. Ralf, and A. J. Smola, "A generalized representer theorem", Int. Conf. on Comput. Learning Theory, vol. 2111, Berlin, Springer, Heidelberg, pp. 416-426, 2001 (DOI: 10.1007/3-540-44581-1_27).

[34] K. Slavakis, B. Pantelis, and S. Theodoridis, "Online learning in reproducing kernel Hilbert spaces", Signal Process. Theory and Machine Learning, pp. 883-987, 2013 (DOI: 10.1016/B978-0-12-396502-8.00017-6)

[35] D. P. Wemerson, C. M. B. Jose, R. Cédric, and T. Jean-Yves, "Stochastic behavior analysis of the Gaussian kernel least-meansquare algorithm", IEEE Trans. on Signal Process., vol. 60, no. 5, pp. 2208-2222, 2012 (DOI: 10.1109/TSP.2012.2186132).

[36] M. Kallas, "Méthodes à noyaux en reconnaissance de formes, prédiction et classification. Applications aux biosignaux", Ph.D. dissertation. Université de Technologie de Troyes, 2012 [Online]. Available: https://hal.archives-ouvertes.fr/tel-01088936 [in French].

[37] W. Liu, P. P. Pokharel, and J. C. Principe, "The kernel least-meansquare algorithm”, IEEE Trans. on Signal Process., vol. 56, no. 2, pp. 543-554, 2008 (DOI: 10.1109/TSP.2007.907881).

[38] P. Kumari and R. Wadhvani, "Wind Power Prediction Using KLMS Algorithm", in Int. Conf. on Inventive Res. in Comput. App. (ICIRCA), Coimbatore, India, pp. 154-161, 2018 (DOI: 10.1109/ICIRCA.2018.8597419).

[39] K. Li and C. P. Jose, "No-trick (treat) kernel adaptive filtering using deterministic features", arXiv preprint arXiv:1912.04530, 2019 [Online]. Available: https://arxiv.org/pdf/1912.04530

[40] J. Ahmed, and K. A. Hamdi, "Spectral efficiency of asynchronous MC-CDMA with frequency offset over correlated fading", IEEE Trans. On Vehicular Technol., vol. 62, no. 7, pp. 3423-3429, 2013 (DOI: 10.1109/TVT.2013.2253339)

[41] M. Zidane and R. Dinis, "A new combination of adaptive channel estimation methods and TORC equalizer in MC-CDMA systems", Int. J. of Commun. Systems, vol. 33, no. 11, pp. e4429, 2020 (DOI: 10.1002/dac.4429).
[42] M. S. Sakib and M. R. Ferdous, "Performance evaluation of MCCDMA system for the next generation wireless networks", in Int. Conf. on Innovation in Engin. and Technol. (ICIET), Dhaka, Bangladesh, pp. 1-5, 2018 (DOI: 10.1109/CIET.2018.8660783).

[43] S. Safi, M. Frikel, A. Zeroual, and M. M'Saad, "Higher order cumulants for identification and equalization of multicarrier spreading spectrum systems", J. of Telecommun. and Informat. Technol., vol. 2, no. 1, pp. 74-84, 2011 [Online]. Available: https://www.itl.waw.pl/czasopisma/JTIT/2011/2/74.pdf

[44] V. Le Nir, J. M. Auffray, M. Hélard, J. F. Hélard, and R. Le Gouable, "Combination of Space-Time Block Coding with MC-CDMA Technique for MIMO systems with two, three and four transmit antennas", IST Mobile \& Wireless Commun. Summit, Aveiro, Portugal, 2003 [Online]. Available: https://hal.archives-ouvertes.fr/ hal-00005810/document

[45] S. Nobilet, "Etude et optimisation des techniques MC-CDMA pour les futures générations de systemes de communications hertziennes". Doctoral dissertation, INSA de Rennes, 2003 [Online]. Available: https://tel.archives-ouvertes.fr/tel-00004081/document [in French].

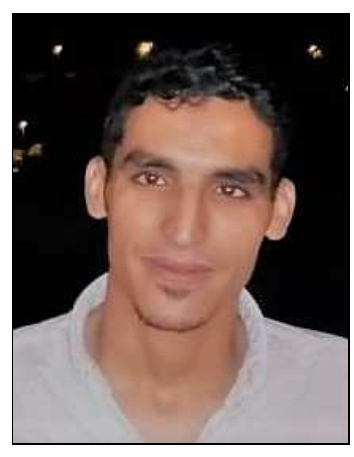

Rachid Fateh received his B.Sc. in Mathematics, Computer Science and Applications from Ibn Zohr University, Ouarzazate, Morocco in 2017 and M.Sc. in Telecommunications Systems and Computer Networks from Sultan Moulay Slimane University, Beni Mellal, Morocco in 2019. $\mathrm{He}$ is pursuing his $\mathrm{Ph} . \mathrm{D}$. in Mathematics and Computer Science from Sultan Moulay Slimane University, Beni Mellal, Morocco. His research interests include system identification, equalization of channel communications with a reproducing kernel Hilbert space, as well as signal processing and estimation.

(iD) https://orcid.org/0000-0002-0574-2105

E-mail: fateh.smi@gmail.com

Laboratory of Innovation in Mathematics, Applications and Information Technologies (LIMATI)

Multidisciplinary Faculty

Sultan Moulay Slimane University

Beni Mellal, Morocco

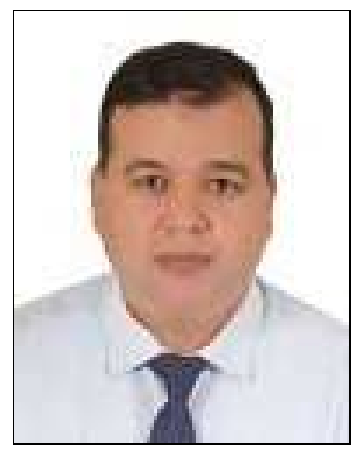

Anouar Darif received his B.Sc. in IEEA (Informatique Electrothecnique, Electronique and Automatique) from Dhar El Mahraz Faculty of Sciences at Mohamed Ben Abdellah University Fez, Morocco in 2005. He received the Diplôme d'Etudes Supérieurs Approfondies in Computer Sciences and Telecommunications from Faculty of Sciences in Rabat in 2007. He received his $\mathrm{Ph} . \mathrm{D}$. degree in Computer Sciences and Telecommunications from the Faculty of Sciences of Rabat in 2015. $\mathrm{He}$ is currently a Research and a Teaching Associate at the Multidisciplinary Faculty at University of Sultan 
Moulay Slimane Beni Mellal, Morocco. His research interests include wireless sensor networks (WSN), mobile edge computing (MFC), Internet of Things (IoT) and Telecommunications (channel identification, IR-UWB, WCDMA, LTE, LTE-A,...).

(iD) https://orcid.org/0000-0001-8026-9189

E-mail: anouar.darif@gmail.com

Laboratory of Innovation in Mathematics, Applications and Information Technologies (LIMATI)

Multidisciplinary Faculty

Sultan Moulay Slimane University

Beni Mellal, Morocco

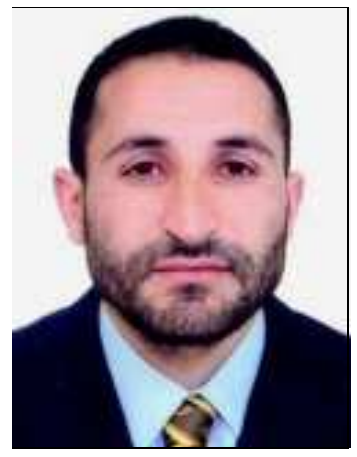

Said Safi received his B.Sc. degree in Physics (option: Electronics) from Cadi Ayyad University, Marrakech, Morocco in 1995, M.Sc. and Ph.D. from Chouaib Doukkali University and Cadi Ayyad University, in 1997 and 2002, respectively. He worked as a Professor of information theory and telecommunication systems at the
National School for Applied Sciences, Tangier, Morocco, from 2003 to 2005. Since 2006, he has been a Professor of applied mathematics and programming at the Multidisciplinary Faculty, Sultan Moulay Slimane University, Beni Mellal, Morocco. In 2008, he received his Ph.D. degree in Telecommunication and Informatics from the Cadi Ayyad University. In 2015, he received the degree of Professor of Sciences at Sultan Moulay Slimane University. His general interests span the areas of communications and signal processing, estimation, time-series analysis, as well as system identification. Safi has published more than 50 journal papers and more than 70 conference papers concerned with the aforementioned topics. His research focuses currently on transmitter and receiver diversity techniques for single- and multi-user fading communication channels, as well as on broad band wireless communication systems.

(iD) https://orcid.org/0000-0003-3390-9037

E-mail: safi.said@gmail.com

Laboratory of Innovation in Mathematics, Applications and Information Technologies (LIMATI)

Multidisciplinary Faculty

Sultan Moulay Slimane University

Beni Mellal, Morocco 